\title{
Community Pharmacists' Involvement in Breast Cancer Health Promotion in United Arab Emirates (UAE)
}

\author{
${ }^{1,2}$ Osama H. Mohamed Ibrahim and ${ }^{1}$ Rana M. Ibrahim \\ ${ }^{1}$ Department of Pharmacy Practice and Pharmacotherapeutics, \\ College of Pharmacy, Sharjah, United Arab Emirates \\ ${ }^{2}$ Department of Clinical Pharmacy, College of Pharmacy, Cairo, Cairo University, Egypt
}

Received 2013-10-09, Revised 2013-10-20; Accepted 2013-10-29

\begin{abstract}
Breast cancer is the most common cancer in women in Middle East countries. Despite the continuous efforts to increase the public awareness of breast cancer via campaigns and public screening programs, breast cancer screening rate remains low. The participation of community pharmacists in the communication and dissemination of breast cancer screening information should have a significant positive impact. The objectives of this study were to assess the degree of community pharmacists' participation in breast cancer health promotion activities in United Arab Emirates (UAE), to evaluate their attitudes towards the involvement in breast cancer health promotion and receiving breast cancer continuous education. The study objectives were addressed in a cross-sectional survey distributed to community pharmacists in Sharjah, Dubai and Ajman Emirates (UAE). The survey measured the extent of community pharmacists' participation in breast cancer health promotion activities, their interest and comfort in providing breast cancer health promotion, and their perceived barriers for integrating breast cancer health promotion activities into their daily practice. Over a 24-week period, we collected 275 surveys out of 335 pharmacists approached ( $82 \%$ response rate). Ninety-six percent indicated that they never invited healthcare professionals to provide breast cancer education in the pharmacy, 67\% said that they never distributed breast cancer educational materials and $47 \%$ reported that they never counseled patients about breast cancer. Nevertheless, more than $75 \%$ were highly interested in being engaged in breast cancer health promotion activities. In addition, $87 \%$ believed that discussing breast cancer awareness with female patients in the pharmacy was beneficial to patients. Yet pharmacists perceived many barriers for integrating breast cancer health promotion into their daily practice including lack of educational materials $(87 \%)$ and lack of enough time (74\%). Moreover, their breast cancer knowledge mean score was $51 \%$ with $87 \%$ expressing a high interest in receiving breast cancer continuous education. Despite their low participation in breast cancer health promotion, the mainstream of pharmacists was interested in educating patients about breast cancer. However, low breast cancer knowledge and other barriers can prevent actualizing this role.
\end{abstract}

Keywords: Community Pharmacists, Breast Cancer, Health Promotion, United Arab Emirates

\section{INTRODUCTION}

Breast cancer is the second most common cancer in the world today and most frequent malignant neoplasm occurring in women. Several studies have shown that breast cancer is the highest incident cancer among women in the Gulf Cooperation Council (GCC) countries (Laliberte et al., 2012). According to the Gulf Center for Cancer Registration, cancer of the breast was by far the most common major malignancy among

Corresponding Author: Osama H. Mohamed Ibrahim, Department of Pharmacy Practice and Pharmacotherapeutics, College of Pharmacy, Sharjah, United Arab Emirates 
females, ranging from $16.1 \%$ of female cancers in Oman to $35.4 \%$ in Bahrain. Overall, only $39(0.9 \%)$ cases $(2$ UAE, 3 Bahrain, 28 Saudi Arabia and 6 Oman) occurred in women aged $<25$ years. However, $57.3 \%$ cases were in women aged 25-50 years at diagnosis. The Age Specific Rate (ASR) per 100000 population was highest in Bahrain (46.4), followed by Kuwait (44.3), Qatar (35.5), UAE (19.2), Oman (14.4) and Saudi Arabia (12.9) (Ravichandran and Zahrani, 2009). Bahrain, Kuwait and Qatar are classified as high- incidence countries and UAE, Saudi Arabia and Oman are lowincidence countries. Due to minimal awareness and cultural taboos, breast cancer mortality rates in UAE are significantly higher than rates in US and UK. UAE joined other nations across the globe in October 2009 in the battle against breast cancer. Two factors make UAE's actions most notable. The first is the high mortality rate associated with breast cancer and the second are the cultural taboos which deter women from coming forward for early diagnosis and screening. Indeed early diagnosis and regular testing are the cornerstones for breast cancer awareness campaigns throughout UAE. Currently, breast cancer is the most common cancer afflicting both Emirati and expat women alike. Twenty-eight percent of all female deaths in UAE are from breast cancer, making it the second most common cause of death. Additionally, forty-four percent of women diagnosed in UAE with breast cancer die from the disease (Camilloni et al., 2013). Early screening for breast cancer saves patients' breasts and increases their chances of complete recovery. Public awareness about breast cancer should be improved as well as screening and early breast cancer detection shall be emphasized (Beshir and Hanipah, 2012).

Pharmacists can easily provide female patients information regarding mammography and education regarding the recommendations in order to encourage increased screening for breast cancer. Pharmacists as health care educators can provide patients information regarding preparation for mammogram and expectations for the procedure. In the community pharmacy setting, further information related to risk factors for breast cancer can be provided to female patients. Pharmacists can notify patients with a family history of breast cancer of the availability of genetic screening and of the recommendations for who should be screened. Community pharmacists can play a crucial role in the early screening and detection of breast cancer by providing educational pamphlets at the pharmacy counter and dropping flyers into prescription bags. They can select an educational day to show videos and display posters for patients explaining the risk factors for breast cancer and the methods for breast self-examination. They can also present with extremely valuable information concerning the symptoms of breast cancer that a female patient should watch for. Pharmacists can invite other health care professionals to provide breast cancer promotional activities and can be engaged in referring patients to breast screening programs (Eades et al., 2011).

Despite the continuous efforts to increase breast cancer public awareness via campaigns and public screening programs, breast cancer screening rate remains low (Hadi et al., 2010). This proves that there is deficiency in the knowledge about the importance of breast cancer early detection among women in UAE. There are many community pharmacies available all over the UAE who can be engaged in the communication and distribution of breast cancer screening information. To our understanding, there are no available studies examining the practices of community pharmacists in breast cancer health promotion in UAE. Furthermore, data are lacking when it comes to pharmacists' attitudes toward breast cancer health promotion and knowledge about the disease (Sadler et al., 2012).

\subsection{Aim of the Study}

The Particular aims of this study were to assess the degree of community pharmacists' participation in breast cancer health promotion activities in United Arab Emirates (UAE), to evaluate their attitudes towards the involvement in breast cancer health promotion and receiving breast cancer continuous education, to illustrate their perceived barriers for integrating into their daily practice breast cancer health promotion activities.

\section{MATERIALS AND METHODS}

\subsection{Study Design, Setting and Participants}

The study objectives were addressed in a crosssectional survey distributed to community pharmacists in Sharjah, Dubai and Ajman Emirates (UAE). The entitled participants were all practicing pharmacists in community pharmacies in UAE.

\subsection{Assessment Tool}

The literature was reviewed by the study investigators who could not detect any published surveys defining the attitudes of community pharmacists in UAE towards involvement in breast cancer health promotion or determining their extent of contribution in breast cancer health promotion activities. The study investigators ended up designing 
the study survey. Breast cancer knowledge questions were articulated according to the American Cancer Society breast cancer early detection guidelines (ACS, 2012). Other questions were designed based on previous surveys (El Hajj and Hamid, 2013).

Once assimilated, the study survey was distributed to 5 highly experienced pharmacists to gauge its appearance validity and readability. Minor modifications were performed based on the obtained feedback. The survey was also pretested among a group of community pharmacists for precision, ease of survey administration and time to completion. The pharmacists self-administered the survey for a more accurate judgment of the contents. Before distributing the final survey to the population, minor improvements were implemented.

The final designed survey contained 28 open and close-ended questions that would be completed within an average of $15 \mathrm{~min}$. The survey enclosed items that addressed community pharmacists' socio-demographic and pharmacy practice characteristics including age, gender, years of experience, average daily prescription volume in the pharmacy, basic qualifications and other relevant information (Table 1). It also included outcomes such as the extent of community pharmacists' participation in breast cancer health promotion activities, the community pharmacists' interest and comfort in providing breast cancer health promotion, their breast cancer knowledge, their interest in receiving breast cancer continuous education, their attitudes and beliefs towards breast cancer health promotion and their perceived barriers for integrating breast cancer health promotion activities into their daily practice. Participants' current involvement in breast cancer health promotion activities was assessed by listing 5 breast cancer health promotion related items (Table 2) and asking participants to specify how often they implemented each activity on a 5-point likert scale (daily, weekly, monthly, every 2 or more months, never). A 3-point scale (high, medium, low) was used to measure participants' interest and comfort in providing breast cancer health promotion and to assess their interest in receiving breast cancer continuous education as explained in (Fig. 1-3). Twelve true or false questions were used to gauge participants' breast cancer knowledge (Table 3). The degree to which pharmacists approved positive statements concerning their role in breast cancer health promotion was measured using a 5point Likert scale (strongly agree, agree, neutral, disagree, strongly disagree) (Table 4). Participants' perceived barriers for integrating breast cancer health promotion were recognized by indicating 11 suggestedbarriers. Pharmacists were asked to indicate on a 5-point
Likert scale the degree to which these constraints would prevent them from incorporating breast cancer health promotion into daily practice (Table 5).

\subsection{Survey Implementation}

Over a 24-week period, 335 surveys were distributed to community pharmacists across UAE (Sharjah, Dubai and Ajman). Three newly graduated pharmacists were assigned this duty and each one handled one area of the UAE. The surveys were disseminated and collected by personal interview. The newly graduated pharmacists randomly selected the pharmacies from the UAE phone directory. The newly graduated pharmacists gave the survey to the community pharmacists and waited to collect the filled survey. The answers of the surveys were entered in a specially designed spread sheet.

Table 1. Socio-demographic and practice characteristics of participated pharmacists

\begin{tabular}{ll}
\hline Characteristic & Frequency $(\%)$ \\
\hline Age $($ years $)(\mathrm{N}=275)$ & \\
$<30$ & $98(36)$ \\
$30-39$ & $112(41)$ \\
$40-50$ & $53(19)$ \\
$>50$ & $12(4)$ \\
Gender $(\mathrm{N}=275)$ & \\
Male & $142(52)$ \\
Female & $133(48)$ \\
Nationality $(\mathrm{N}=275)$ & \\
Egypt & $113(41)$ \\
India & $57(21)$ \\
Philippines & $45(16)$ \\
Jordan & $41(15)$ \\
Other middle Eastern countries & $19(7)$ \\
Number of years since pharmacy & \\
graduation $(\mathrm{N}=275)$ & \\
$<5$ & $76(28)$ \\
$6-10$ & $89(32)$ \\
$11-15$ & $59(21)$ \\
$16-20$ & $41(15)$ \\
$>20$ & $10(4)$ \\
Highest pharmacy degree $(\mathrm{N}=275)$ & \\
Bachelor of pharmacy & $243(88)$ \\
Pharm D & $18(7)$ \\
MSc & $14(5)$ \\
Average daily prescription & \\
volume in the pharmacy $(\mathrm{N}=275)$ & \\
$<50$ & $146(53)$ \\
$50-99$ & $85(31)$ \\
$>100$ & $44(16)$ \\
Percentage of female patients & \\
seen in the pharmacy per day $(\mathrm{N}=275)$ & $96(35)$ \\
$<25 \%$ & $110(40)$ \\
$25-50$ & $46(17)$ \\
$51-75$ & $23(8)$ \\
$76-100$ & \\
\hline & \\
&
\end{tabular}


Table 2. Participants' current involvement in breast cancer health promotion activities

\begin{tabular}{|c|c|c|c|c|c|}
\hline \multirow[b]{2}{*}{ Statement } & \multicolumn{5}{|c|}{ Frequency $(\%)$} \\
\hline & Daily & Weekly & Monthly & $\begin{array}{l}\text { Every } 2 \text { or } \\
\text { more months }\end{array}$ & Never \\
\hline \multicolumn{6}{|l|}{ On average how often do you do the following? } \\
\hline $\begin{array}{l}\text { Respond to patient inquiries related to breast cancer warning signs } \\
\text { and symptoms and breast cancer early detection and screening tests }\end{array}$ & $8(3)$ & $31(11)$ & $54(20)$ & $78(28)$ & 104(38) \\
\hline $\begin{array}{l}\text { Provide patients with advice or counseling on breast cancer } \\
\text { screening and early detection }\end{array}$ & $3(1)$ & $6(2)$ & $57(21)$ & $79(29)$ & $130(47)$ \\
\hline $\begin{array}{l}\text { Provide patients with breast cancer educational materials or } \\
\text { self-assessment quizzes }\end{array}$ & $3(1)$ & $8(3)$ & $34(12)$ & $45(16)$ & $185(67)$ \\
\hline $\begin{array}{l}\text { Invite healthcare professionals to provide breast } \\
\text { cancer education to patients in the pharmacy }\end{array}$ & $0(0)$ & $1(<1)$ & $3(1)$ & $8(3)$ & $263(96)$ \\
\hline $\begin{array}{l}\text { Refer patients to special breast cancer screening programs organized } \\
\text { by hospitals or cancer organizations in United Arab Emirates }\end{array}$ & $3(1)$ & $8(3)$ & $65(24)$ & $78(28)$ & $121(44)$ \\
\hline
\end{tabular}

Table 3. Participants' performance on breast cancer knowledge items

Breast cancer knowledge items

Breast cancer is the most common form of cancer among women Breast cancer should not be of concern for patients younger than 40 years of age Use of hormone replacement therapy is one of the risk

factors for developing breast cancer

Late onset menstrual period is one of the risk factors for developing breast cancer

Nipple discharge can be a warning sign of breast cancer

Appropriate early screening for breast cancer reduces breast cancer mortality

Breast self-exam is one of the methods that are used to

detect the presence of breast cancer

To detect the presence of breast cancer, women over the age of 20 and under the age of 40 should do a breast self-exam at least once per year To detect the presence of breast cancer, women aged 40 years and above should do a monthly breast self-exam, an annual clinical

breast exam and a biannual mammogram

Total mastectomy is the surgical option of choice for patients diagnosed with early stage breast cancer

Patients with invasive breast cancer that is estrogen receptor positive

should receive adjuvant endocrine therapy

Tamoxifen is the adjuvant endocrine therapy of choice for premenopausal patients with invasive breast cancer

\begin{tabular}{llll} 
(Percent) & & & \\
- Correct & & & \\
answer & Correct & Incorrect & $\begin{array}{l}\text { Did not } \\
\text { know }\end{array}$ \\
True & $(92)$ & $(5)$ & $(3)$ \\
False & $(69)$ & $(13)$ & $(18)$ \\
True & $(87)$ & $(10)$ & $(3)$ \\
False & $(37)$ & $(38)$ & $(25)$ \\
True & $(65)$ & $(13)$ & $(22)$ \\
True & $(96)$ & $(2)$ & $(2)$ \\
True & $(97)$ & $(2)$ & $(1)$ \\
False & $(34)$ & $(59)$ & $(7)$ \\
& & & \\
False & $(22)$ & $(68)$ & $(10)$ \\
False & $(55)$ & $(36)$ & $(9)$ \\
True & $(58)$ & $(12)$ & $(30)$ \\
True & $(84)$ & $(6)$ & $(10)$ \\
\hline & & &
\end{tabular}

\subsection{Data Analysis and Confidentiality}

The database for participants' responses was created in Microsoft Excel and exported into Statistical Package of Social Sciences (SPSS) version 17 for descriptive analysis. Partial surveys were incorporated in the analysis if they contained incomplete answers to some of the questions and other basic demographic data. To simplify analysis of data, five-point Likert scale answers were assembled into three general categories (agree, neutral, disagree) and (high, medium and low). To secure the confidentiality of participants and to reduce the risk of bias, investigators did not require any pharmacy or participant identification information.

\subsection{Ethical Considerations}

Because the study did not pose any risk to participants, they were not required to fill out any formal consent form. Pharmacists' participation in the study was voluntary and that was communicated over the phone when the study objectives were explained to participants. 
Table 4. Participants' attitudes and beliefs towards breast cancer health promotion $(N=275)$ What is your extent of agreement with the following statements?

(Percent)

Statement

I should be involved in breast cancer health promotion activities in the pharmacy

Integrating breast cancer health promotion into my daily practice as a community pharmacist is important

I feel confident and prepared to provide breast cancer health promotion

Discussing breast cancer awareness with my female patients in the pharmacy

is beneficial and can save their lives

Providing breast cancer counseling to my female patients in the pharmacy

is my responsibility as a pharmacist

Distributing breast cancer educational materials is important in the pharmacy

It is important to discuss breast cancer with my female patients to encourage

breast cancer early screening and detection

There is enough evidence to suggest that the pharmacist can influence patients to adopt breast cancer screening practices

Inviting healthcare professionals to provide breast cancer education to the female patients in the pharmacy is important

Providing breast cancer counseling to my patients can improve my professional

state and increase my professional satisfaction

Providing breast cancer counseling is an effective use of my time

If I have access to patient education materials related to breast cancer I am more

likely to provide breast cancer health promotion to my female patients

Patients would like me as a pharmacist to counsel them on breast cancer

screening and early detection

Patients appreciate my effort as a pharmacist to counsel them on breast cancer

Table 5. Participants' perceived barriers for integrating breast cancer health promotion

Barrier

There are not enough personnel

I do not have enough time

I do not have enough space

I do not have breast cancer educational materials

Providing breast cancer health promotion does not interest me

As a pharmacist, I am not supposed to provide breast cancer health promotion

I do not have adequate knowledge or skills in this area

Patients do not appreciate the pharmacist's role as a breast cancer health promoter

Patients will be offended if I offer them breast cancer counseling

I am not reimbursed for providing breast cancer health promotion

My cultural and/or religious beliefs prevent me from providing breast cancer health promotion

\section{RESULTS}

Over a 6 month period, we were able to collect 275 surveys out of 335 pharmacists approached $(82 \%$ response rate). Socio-demographic and practice characteristics of respondents are presented in Table $\mathbf{1 .}$ The percentage of male respondents was slightly higher $(52 \%)$ and the majority of respondents were less than 40 years of age (41\%). Most participants (93\%) were from countries: Egypt, India, Philippines and Jordan. The percentage of female patients seen in the pharmacy per day between the ages of (25-50) was (40\%).

Respondents' involvement in breast cancer health promotion is presented in Table 2. About $47 \%$ of the pharmacists reported that they never provided patients with advice or counseling on breast cancer screening and early detection, $67 \%$ never provided patients with breast cancer educational materials or self assessment 
quizzes and $96 \%$ of them never invited healthcare professionals to provide breast cancer education to patients in the pharmacy.

Figure 1 and 2, illustrate pharmacists' interest and comfort in providing breast cancer health promotion.

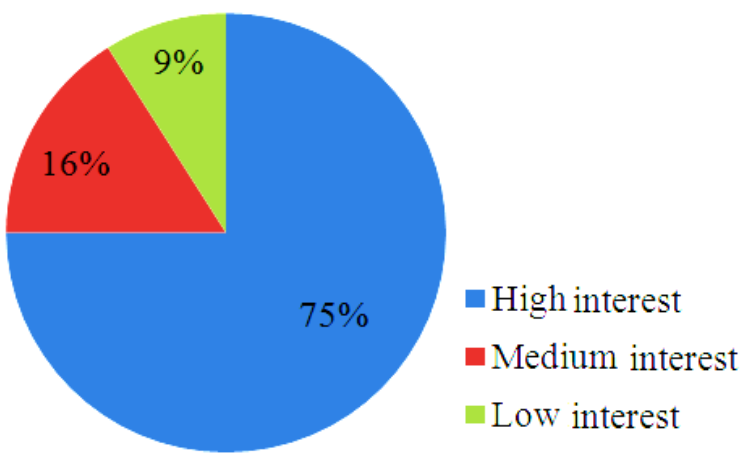

Fig. 1. Interest in providing breast cancer health promotion. $\mathrm{N}$ $=275$ respondents. Responses have been collapsed into a 3-point scale; high, medium and low

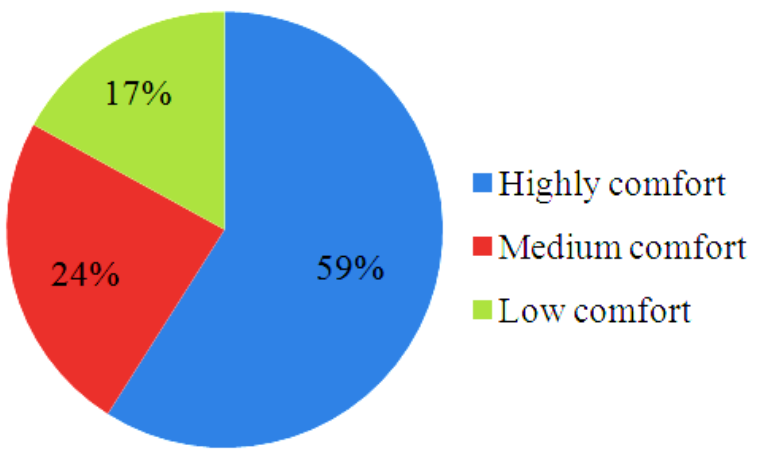

Fig. 2. Comfort in providing breast cancer health promotion. N $=275$ respondents. Responses have been collapsed into a 3-point scale; high, medium and low

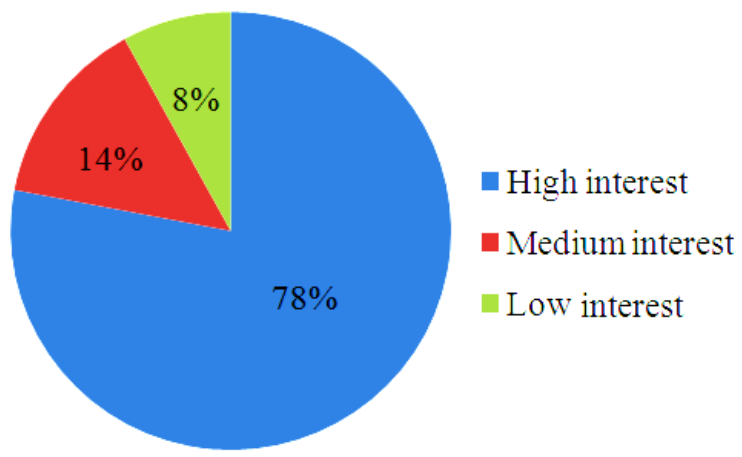

Fig. 3. Interest in receiving breast cancer continuous education. $\mathrm{N}=275$ respondents. Responses have been collapsed into a 3-point scale; high, medium and low
Surprisingly, 206 respondents (75\%) indicated that they were highly interested in providing breast cancer health promotion and 162 respondents (59\%) were highly comfortable in delivering this activity.

Figure 3, shows community pharmacists' interest in receiving breast cancer continuous education. The majority of respondents represented by 215 (78\%) were highly interested in receiving breast cancer continuous education. Participants' performance on breast cancer knowledge items is illustrated in Table 3. Awareness of breast cancer was assessed using twelve true or false cancer related questions. Scores were noticed to be low for questions associated with breast cancer screening recommendations and risk factors.

Table 4 represents the degree of pharmacists' agreement with constructive statements regarding their responsibility towards breast cancer health promotion. Community pharmacists' views highly supported breast cancer education. About $87 \%$ of the participants believed that discussing breast cancer awareness with female patients in the pharmacy is beneficial and can save their lives. Furthermore, $86 \%$ agreed that their professional status and satisfaction can be improved through provision of breast cancer counseling in the pharmacy.

Table 5 shows pharmacists' perceived barriers for providing breast cancer health promotion. Community pharmacists identified many constraints for playing this role. Highly identified barriers included respectively: deficiency in breast cancer educational materials (87\% of participants), lack of time (74\% of participants), insufficient personnel (68\% of respondents) and lack of reimbursement for such services (50\% of participants).

\section{DISCUSSION}

This study explored pharmacists' roles as public health educators, especially in breast cancer health promotion. To date little information has been gathered in UAE on involvement of community pharmacists in provision of breast cancer health promotion services. Our results indicated that few community pharmacists in UAE are engaged in breast cancer health promotion activities. This may indicate a lack of patient's awareness of the type of services that pharmacists can offer, the type of counseling and education pharmacists are capable of delivering, insufficient pharmacists' abilities and knowledge, in addition to the probability that patients may access such health information from other sources and maybe hesitant to discuss it with a pharmacist (Bi Suh et al., 2012). The degree of 
improvement in community pharmacy practice in UAE, as other Middle Eastern countries is slow. Pharmacy practice in UAE is still in the early development phase and pharmacist skills and training appear to be underutilized (El Hajj and Hamid, 2013). Except for very few cognitive and patient oriented activities performed, community pharmacists' job is limited to medication dispensing (Awad and Abahussain, 2010). The absence of a pharmacists' national organization that is dedicated to back up the professional development of pharmacists and to expand the pharmacy profession is one of the contributing factors to this slow improvement in pharmacy practice (Taha et al., 2012). The majority of pharmacists was willing to be involved in educating their patients about breast cancer and viewed breast cancer health promotion as their responsibility, despite their low engagement in breast cancer health promotion activities. Nevertheless, more than $75 \%$ were highly interested in being engaged in breast cancer health promotion activities and $87 \%$ believed that discussing breast cancer awareness with female patients in the pharmacy was beneficial to patients. Community pharmacists in UAE are highly interested in shifting their profession from being drug dispensing centered to patient pharmaceutical care centered. $88 \%$ agreed that there is enough evidence to suggest that the pharmacist can influence patients to adopt breast cancer screening. Most importantly, $86 \%$ agreed that providing breast cancer counseling to their patients improves their professional satisfaction. These results are highly significant considering the goal to improve breast cancer awareness in UAE. Pharmacists are easily accessible and recognized as experts in matters of health; therefore, they could offer public health interventions more conveniently than other health care providers (Park et al., 2011). Furthermore, community pharmacists are the ideal site for credible counseling for a large segment of the population because pharmacists have frequent contact with the public, have extended opening hours and are widely distributed geographically. However, pharmacists working in non-institutionalized settings, such as pharmacies are not being fully utilized (Cook et al., 2011). Pharmacy graduates are well trained to be clinicians; however, community pharmacy practitioners may not be able to use their clinical skills in the community setting. A good question to ask is, "What are the health provider roles of UAE pharmacists in the community?" It seems that UAE pharmacists are not prepared to the role of breast cancer health promoters. Even though the majority of the pharmacists surveyed in our study, indicated that they were comfortable in providing breast cancer health promotion to female patients, their mean breast cancer knowledge score was $51 \%$ with $87 \%$ expressing a high interest in receiving breast cancer continuous education. Still, low breast cancer knowledge in addition to other identified barriers can prevent community pharmacists from actualizing their role in breast cancer education (Boyd et al., 2010).

Current pharmacy education curricula may not sufficiently prepare graduates to take on the role of public health educator within the community. Another point to consider is that, continuous pharmacy education should be offered to UAE's community pharmacists focusing on public health education in many areas including all type of cancers (Sambanje and Mafuvadze, 2012). The University of Sharjah, college of pharmacy added breast cancer continuing education workshops to its agenda, since most of the pharmacists in our study expressed interest in receiving additional breast cancer education.

The community pharmacists cite lack of breast cancer educational materials (87\%), lack of time (74\%) and lack of personnel $(68 \%)$ as major barriers that limit their involvement in breast cancer health promotion activities. Lack of time is an obstacle that stands against the provision of cognitive services by pharmacists worldwide (Jorgenson et al., 2011). About $68 \%$ of the pharmacists reported that they don't have enough personnel. Thus for pharmacists to provide breast cancer health promotion activities without being continuously interrupted by phone calls and patients, more pharmacy technicians need to be hired and there should be a clear defined distinction between the role of the pharmacist and the one of a pharmacy technician.

Government policy, remuneration for service delivery, communication and teamwork, task delegation, reorganization of the function and structure of the pharmacy and patient expectations regarding the services to be offered could serve as facilitators for change in community pharmacy (Krska and Morecroft, 2010). Another issue is that, current prescription volumes in community pharmacy may be an impediment to the provision of health promotion services. Thus, innovative methods are needed for pharmacists to adequately provide cancer information to patients without disruption in their daily activities. The use of touch screen technology in facilitating breast cancer education could be a solution for this matter.

Another perceived barrier was lack of patients' appreciation for the role of the pharmacist as a breast cancer health promoter. This barrier could be overcome 
by educating the public about the role of the pharmacist in cancer health promotion and encourage UAE's pharmacists to promote their capabilities in this area.

Another barrier was lack of breast cancer educational materials. To by-pass this barrier, national cancer organization in UAE should be encouraged to supply community pharmacies with breast cancer educational material for distribution to the public.

In addition, undergraduate pharmacy students in all colleges of pharmacy should be well prepared for breast cancer health promotion (Woloshin et al., 2012).

\subsection{Limitations}

Our study has its limitations. First of all, study variables were assessed by self-report, which may be biased by an inclination to provide socially desirable responses, acquiescence (tendency to agree) and extremity (tendency to use extreme ratings). Another limitation is that the survey reliability was not tested among the population of UAE's community pharmacists. Finally, the survey sample size was relatively small. Thus, generalization of the study results to all UAE's pharmacists should be done cautiously.

\section{CONCLUSION}

The results from this study suggest that pharmacists are willing to take on the responsibility of health education and risk communication in the prevention and detection of breast cancer. This study represents the first attempt to assess the current breast cancer health promotion practices of community pharmacists in the UAE. Although the results indicated that few community pharmacists are engaged in breast cancer health promotion activities, the majority of pharmacists view breast cancer health promotion as a part of their responsibility. However, low breast cancer knowledge in addition to other identified barriers can prevent actualizing pharmacists' role in breast cancer health promotion. Therefore, additional efforts should be made on providing these pharmacists with high quality breast cancer continuous education and overcoming all identified barriers to allow these pharmacists to make a crucial contribution to breast cancer education in UAE.

\section{ACKNOWLEDGEMENT}

Our sincere gratitude goes to our newly graduated pharmacists for their assistance during data collection of this study. We also acknowledge the community pharmacists for sharing their sincere views and opinions with us.

\subsection{Conflicts of Interest}

The authors of this manuscript have no conflict of interest to declare.

\section{REFERENCES}

ACS, 2012. Breast cancer. American Cancer Society.

Awad, A. and E. Abahussain, 2010. Health promotion and education activities of community pharmacists in Kuwait. Pharm. World Sci., 32: 146-153. DOI: 10.1007/s1 1096-009-9360-6

Beshir, S.A. and M.A. Hanipah, 2012. Knowledge, perception, practice and barriers of breast cancer health promotion activities among community pharmacists in two Districts of Selangor State, Malaysia. Asian Pacific J. Cancer Prevent., 13: 4427-4430. DOI: 10.7314/APJCP.2012.13.9.4427

Bi Suh, M.A., J. Atashili, E.A. Fuh and V.A. Eta, 2012. Breast self-examination and breast cancer awareness in women in developing countries: A survey of women in Buea, Cameroon. BMC Res Notes, 5: 627-627. DOI: 10.1186/1756-0500-5-627

Boyd, N.F., L.J. Martin, M. Bronskill, M.J. Yaffee and N. Duric et al., 2010. Breast Tissue Composition and Susceptibility to Breast Cancer. J. Natl. Cancer Inst., 102: 1224-1237. PMID: 20616353

Camilloni, L., E. Ferroni, B.J. Cendales, A. Pezzarossi and G. Furnari et al., 2013. Methods to increase participation in organised screening programs: A systematic review. BMC Public Health, 13: 464464. DOI: 10.1186/1471-2458-13-464

Cook, W.J., W.H. Cunningham, W.R. Pulleyblank and A. Schrijver, 2011. Combinatorial Optimization. 1st Edn., John Wiley and Sons, New York, ISBN-10: 1118031393 , pp: 368.

Eades, C.E., J.S. Ferguson and R.E. O'Carroll, 2011. Public health in community pharmacy: A systematic review of pharmacist and consumer views. BMC Publ Health, 11, 582-582. DOI: 10.1186/1471-245811-582

El Hajj, M.S. and Y. Hamid, 2013. Breast cancer health promotion in Qatar: A survey of community pharmacists' interests and needs. Int. J. Clin. Pharmacy, 35: 376-385. DOI: 10.1007/s11096-0109449-y

Hadi, M.A., M.A. Hassali, M.A. Shafie and A. Awasiu, 2010. Evaluation of breast cancer awareness among female university students in Malaysia. Pharmacy Pract., 8: 29-34. 
Jorgenson, D., D. Lamb and N.J. MacKinnon, 2011. Practice change challenges and priorities: A national survey of practising pharmacists. Can. Pharm. J., 144: 125-131. DOI: 10.3821/1913-701X-144.3.125

Krska, J. and C.W. Morecroft, 2010. Views of the general public on the role of pharmacy in public health. J. Pharm. Health Serv. Res., 1: 33-38. DOI: 10.1211/jphsr.01.01.0013

Laliberte, M.C., S. Perreault, N. Damestoy and L. Lalonde, 2012. Ideal and actual involvement of community pharmacists in health promotion and prevention: A cross-sectional study in Quebec, Canada. BMC Public Health, 12: 192-192. DOI: 10.1186/1471-2458-12-192

Park, K., W.H. Hong, S.Y. Kye, E. Jung and M.H. Kim et al., 2011. Community-based intervention to promote breast cancer awareness and screening: The Korean experience. BMC Public Health, 11: 468468. DOI: $10.1186 / 1471-2458-11-468$

Ravichandran, K. and A.S. Zahrani, 2009. Association of reproductive factors with the incidence of breast cancer in gulf cooperation council countries. East. Mediterr Health J., 15: 612-621. PMID: 19731777
Sadler, G.R., P.R. Beerman, K. Lee, J. Hung and H. Nguyen et al., 2012. Promoting breast cancer screening among Asian American women: The Asian grocery store-based cancer education program. J. Cancer Educ., 27: 612-617. DOI: 10.1007/s13187-012-0419-z

Sambanje, M.N. and B. Mafuvadze, 2012. Breast cancer knowledge and awareness among university students in Angola. Pan. Afr. Med. J., 11: 70-70. PMID: 22655104

Taha, H., R. Al-Qutob, L. Nystrom, R. Wahlstrom and V. Berggren, 2012. "Voices of fear and Safety" Women's ambivalence towards breast cancer and breast health: A qualitative study from Jordan. BMC Womens Health, 12: 21-21. DOI: 10.1186/14726874-12-21

Woloshin, S., L.M. Schwartz, W.C. Black and B.S. Kramer, 2012. Cancer screening campaigns-getting past uninformative persuasion. New Engl. J. Med., 367: 1677-1679. DOI: 10.1056/NEJMp1209407 